\title{
Single-crystal sapphire microstructure for high-resolution synchrotron x-ray monochromators
}

\author{
Victor E. Asadchikov ${ }^{1,2}$, Andrey V. Butashin ${ }^{1}$, Alexey V. Buzmakov ${ }^{1}$, Alexander N. Deryabin $^{1}$, \\ Vladimir M. Kanevsky ${ }^{1}$, Igor A. Prokhorov ${ }^{3}$, Boris S. Roshchin ${ }^{1}$, Yuri O. Volkov ${ }^{1}$, Denis A. Zolotov ${ }^{1}$, \\ Atefeh Jafari, ${ }^{4,5,6}$, Pavel Alexeev ${ }^{4,7}$, Angelica Cecilia ${ }^{8}$, Tilo Baumbach ${ }^{8}$, Dimitrios Bessas ${ }^{6}$, Andreas N. \\ Danilewsky ${ }^{9}$, Ilya Sergueev ${ }^{7}$, Hans-Christian Wille ${ }^{7}$, Raphaël P. Hermann ${ }^{*}, 4,5,10$
}

Received December xx 2015, revised zzz, accepted zzz,

Published online zzz

Keywords $\mathrm{x}$-ray optics, topography, sapphire, dislocations

We report on the growth and characterization of sapphire single crystals for $\mathrm{x}$-ray optics applications. Structural defects were studied by means of laboratory double-crystal X-ray diffractometry and white-beam synchrotron-radiation topography. The investigations confirmed that the main defect types are dislocations. The best quality crystal was grown using the Kyropoulos technique. Therein the dislocation density was $10^{2}-10^{3} \mathrm{~cm}^{-2}$ and a small area with approximately $2 * 2 \mathrm{~mm}^{2}$ did not show dislocation contrast in many reflections. This crystal has suitable quality for application as a backscattering monochromator. A clear correlation between growth rate and dislocation density is observed, though growth rate is not the only parameter impacting the quality.

\section{Introduction}

The appearance of modern third-generation synchrotron and free-electron laser X-ray sources with ultra-high brilliance and significant coherence places strict demands on X-ray optics. In many cases $\mathrm{X}$-ray optics become a limiting factor for utilization of these powerful sources. Potential problems include heat-load management [1], precision focusing (by e.g. multilayer grazing-incidence mirrors or bent crystals) [2] and monochromatization by e.g. crystal monochromators [3] or gratings [4].
Specifically, nuclear resonance scattering requires ultra-narrow bandpass probe beams both to reduce the photon load on the detector and to resolve the nuclear inelastic scattering. For example, nuclear inelastic scattering at energies higher than $30 \mathrm{keV}$ requires both a relative energy resolution and a beam energy control of better than $\Delta \mathrm{E} / \mathrm{E}=3.10^{-8}$ in order to achieve a resolution of $1 \mathrm{meV}$.

This extreme level of energy precision can be achieved with the use of high-order Bragg reflections. However, the spectral reflectivity factor must be high (at least 10\%) while the divergence of the incident synchrotron radiation beam should not exceed the

* Corresponding author: hermannrp@ornl.gov, +1 8655764264

${ }^{1}$ Shubnikov Institute of Crystallography RAS, 119333 Leninskii pr-t 59, Moscow, Russia

2 Lomonosov Moscow State University, Faculty of Physics, 119991, GSP-1, 1-2 Leninskiye Gory, Moscow, Russia

${ }^{3}$ Research Center for Space Materials Science, Kaluga Branch of Shubnikov, Institute of Crystallography RAS, Kaluga, 248640 Russia

${ }^{4}$ Jülich Centre for Neutron Science JCNS and Peter Grünberg Institute PGI, Forschungszentrum Jülich, 52425 Jülich, Germany

${ }^{5}$ Faculty of Sciences, University of Liège, 4000 Liège, Belgium

${ }^{6}$ European Synchrotron Radiation Facility, 38043 Grenoble, France

${ }^{7}$ Deutsches Elektronen-Synchrotron, 22607 Hamburg, Germany

${ }^{8}$ Karlsruhe Institute of Technology, Institute for Photon Science and Synchrotron Radiation and ANKA Synchrotron Radiation Facility, 76344 Eggenstein-Leopoldshafen, Germany

${ }^{9}$ Crystallography Institute of Geo- and Environmental Sciences, AlbertLudwigs University Freiburg, 79104 Freiburg, Germany

10 Materials Science and Technology Division, Oak Ridge National Laboratory, Oak Ridge, 37831 Tennessee, USA 
angular width of the corresponding Bragg reflection for the chosen crystal. Both requirements can be fulfilled for a variety of asymmetrically cut crystals for radiation energies less than $30 \mathrm{keV}$ [5]. At higher energies, this approach becomes ineffective due to geometric limitations and reflectivity factor loss. Hence, an alternative monochromatization scheme based on a single high order Bragg reflection in socalled backscattering geometry is then used [6]. In this scheme a precise energy selection is obtained by a temperature-dependent change in $d$-spacing while the Bragg reflection angle remains as close as possible to $90^{\circ}$. The implementation of this scheme requires a cryostat housing for the monochromator crystal with a high-precision and high-stability temperature control -of the order of a few $\mathrm{mK}$ - providing a meV resolution for scanning the radiation energy.

For the selection of Bragg reflections close to the fixed desired nuclear resonance energy in the 100-400 K temperature range, crystals with cubic symmetry generally provide an insufficient density of reflections. Thus single-crystalline silicon, despite its wide applicability to X-ray crystal optics and the availability of high-quality crystals, does not meet the requirements for this particular application. One of the best alternatives is single-crystalline corundum white sapphire [7] first utilized for back scattering monochromatization purpose at $25.6 \mathrm{keV}$ at DESY [8]. The sapphire crystal structure belongs to the rhombohedral system and exhibits a high density of Bragg back-reflections. Furthermore, its melting temperature of $2050^{\circ} \mathrm{C}$ largely exceeds that of silicon, a first indication of a higher Debye temperature and thus higher Debye-Waller factor, $D W F=\exp (-$ $<(\mathbf{q} . \mathbf{u})^{2}>$ ), where $\mathbf{q}$ is the scattering vector and $\mathbf{u}$ the atomic displacement vector. Such a high DebyeWaller factor is needed in order to achieve high X-ray spectral reflectivity. Current growth technologies allow the growth of nearly perfect sapphire single crystals [9]. Despite the higher density of defects than in single-crystalline silicon, the half-width of the corresponding diffraction reflectivity curves is narrower but still exceeds the theoretical one. Current sapphire polishing technology provides a superior level of surface quality (i.e. surface roughness values) in comparison to any other known crystalline materials including silicon [10]. It is also possible to form specific surface nano-reliefs which facilitate the subsequent deposition of thin film coatings [11]. Note that the formation of such regular nano-reliefs could also yield a selective increase in reflectivity [12].
First results on the application of the sapphire backscattering monochromatization approach above $30 \mathrm{keV}$ have been obtained at the European Synchrotron Radiation Facility (ESRF). An energy resolution of approximately $0.7-1.2 \mathrm{meV}$ has been achieved, with monochromatization efficiencies significantly higher than for silicon based setups $[13,14]$ but still suboptimal. In view of these promising results, the goal of the presented work is to obtain an understanding on the optimized process for the fabrication of crystalline monochromators based on synthetic sapphire crystals of high perfection, notably in terms of crystal quality as a function of the growth technique.

The sample used in Ref. [13] had an area of only $300 \times 300 \mu \mathrm{m}^{2}$ which could provide the desired energy resolution of $<1 \mathrm{meV}$. Hence, in this work we propose a comparative analysis of the real structure of sapphire crystals synthesized by the Verneuil, Czochralski, Kyropoulos-Musatov, and Horizontallydirected solidification techniques $[9,10]$ in order to later optimize the structural quality for the fabrication of x-ray optics elements.

Laboratory X-ray topography methods, rocking curve analysis, and synchrotron radiation topography will be applied to the investigation of structural imperfections in the crystals. The X-ray diffraction methodology is based on rocking curve scanning, i.e. angular dependencies of the X-ray beam intensity reflected by a specific set of atomic planes, measured within the small range of angular positions that meets the Bragg condition of the crystal. Values that define the experimental rocking curve profile such as fullwidth at half-maximum, integral, and peak intensity allow for qualitative determination of crystalline perfection, while comparison of the experimental versus numerically modeled rocking curves allows for the detection of structural changes within the investigated sample. Topography by the Lang method and synchrotron white beam topography yield direct images of crystalline defects with high spatial resolution and are thus methods of choice for this study.

\section{Experimental}

In order to reveal the factors that limit the quality of sapphire crystals and to select the best wafers, a set of 15 crystals was grown by different techniques. The crystals were grown by the Kyropoulos method -in which the seed is cooled and the melt temperature 
reduced-, Horizontally-directed solidification method (HDSM), Bridgman, and Verneuil techniques [9, 12], with pulling rates of $0.25-1,6-10,2-5$, and $10-15$ $\mathrm{mm} / \mathrm{h}$, respectively, and a diameter for the grown boules of approximately 90, 90, 20 and $300 \mathrm{~mm}$ and lengths of $120,200,100$, and $200 \mathrm{~mm}$, respectively. Double-sided wafers with approximate dimensions of $10 \times 10 \times 1 \mathrm{~mm}^{3}$ were cut from the as grown samples. All wafers were chemically polished on both sides, and the wafers grown by the Kyropoulos and Verneuil methods were subjected to additional mechanical polishing.

Laboratory X-ray topography was performed with the setup shown in Fig. 1. Investigations by doublecrystal X-ray diffractometry and plane-wave reflection topography were performed on a doublecrystal diffractometer with $\mathrm{CuK} \alpha 1$ radiation and a point detector.

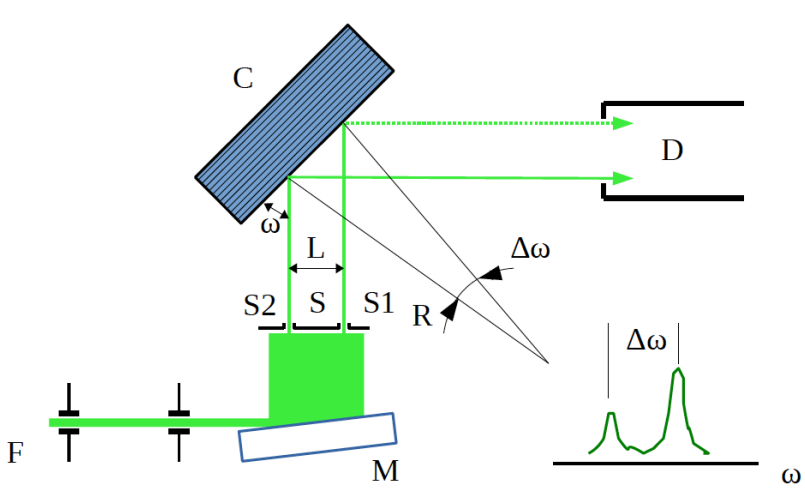

Figure 1: Laboratory setup for X-ray topography: F - X-ray source, M - Ge monochromator crystal, S,S1,S2 - slits, C sample, D - point detector. The double-slit mode enables sample curvature measurements.

The reflections for the investigations are in good interplanar parameter conformity with the 511 reflection in germanium with Bragg angle $\theta=45.07^{\circ}$ and reflection asymmetry factor $b=0.01$ with incident angles $\omega=\theta-\varphi>0$ (here $\theta$ is the Bragg angle and $\varphi$ is the angle of the reflecting atomic planes relative to the sample surface), see Fig. 1. Diffractometry has been performed with a single slit opening.

Double-crystal X-ray topography has been performed with a laboratory source with a wide beam and a $\sim 0.5$ $\mathrm{cm}$ slit. For determination of the wafer curvature radius $R$ a double slit opening was used. For unbent crystals, beams S1 and S2 satisfy the reflection conditions simultaneously. However, in case of a bent crystal two diffraction peaks can be detected during the rotation of the crystal around Bragg axis, the angle between the peaks is proportional to the curve radius as $R=L / \Delta \omega \cdot \sin \omega$ (here $\omega$ is the grazing angle of incident beam at surface, $L$ is the distance between slits $S_{1}$ and $S_{2}$ ). With the setup parameters used here, curvatures of less than $500 \mathrm{~m}$ radius could be detected.

Further, white-beam topography was performed at the TOPO-TOMO beam line at the ANKA synchrotron radiation source, Karlsruhe, Germany (transmission topography configuration; distance sample to film = $8.7 \mathrm{~cm}$; Slavich VRP-M film, grain size $0.05 \mu \mathrm{m}$ - Fig. 2 [15]). We used software package called LauePt [16] to find the corresponding reflections. An example of comparison of simulation and experimentally obtained reflections on a film is presented in Fig. 3.

\section{Detecting film}

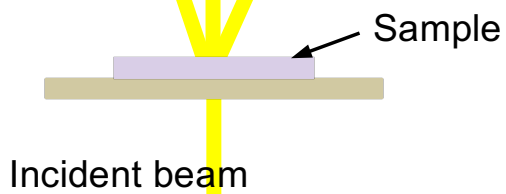

Figure 2: Experimental setup for white beam topography.

Note that the simulation shows reflections in rhombohedral indices. Since it is common to use four indices for sapphire crystals, they are converted into hexagonal index notation for the rest of the report.

Backscattering high-resolution topography $[13,17]$ using an inline monochromator with a bandwidth of $0.7 \mathrm{meV}$ at $23.9 \mathrm{keV}$ was carried at the nuclear resonance beam line P01, Petra III, DESY, Hamburg on the most promising crystal, sample \#1.

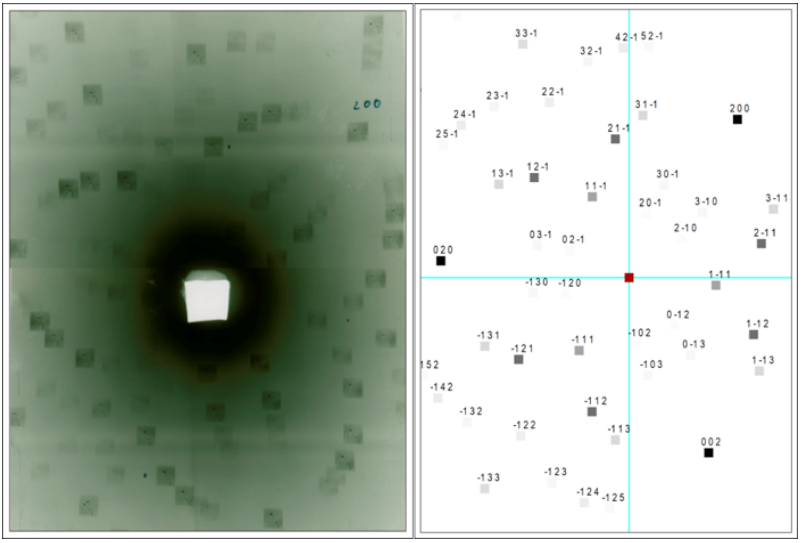

Figure 3 Example of experimental (left) and simulated (right) Laue patterns for wafer \#1. Reflections are indexed in the rhombohedral setting. 


\section{Results}

Herein we show results obtained on five selected wafers as summarized in Table 1. The first four crystals were grown by the Kyropoulos, HDSM, and Verneuil techniques. The fifth sample was a commercial sample cut from a Kyropoulos grown boule at the "Monocrystal" company (Stavropol). Representative topographs of all five wafers are shown and detailed below. Overall only wafer \#1 and small areas in wafer \#5 exhibit a low dislocation density of $10^{3} \mathrm{~cm}^{-2}$ or less and only the defects in wafer \#1 will be discussed in detail.

\begin{tabular}{|l|l|l|l|}
\hline \multicolumn{3}{|c|}{ Table 1 Sapphire single crystal wafer specifications } \\
\hline $\begin{array}{l}\text { No., Name/Diameter } \\
(\mathbf{m m}) \text {,thickness }(\mathbf{m m})\end{array}$ & Orientation & $\begin{array}{l}\text { Growth } \\
\text { technique }\end{array}$ & $\begin{array}{l}\text { Pulling } \\
\text { rate } \\
\text { (mm/h) }\end{array}$ \\
\hline \#1,SK1C/22, 1 & $\left(\begin{array}{llll}0 & 0 & 0 & 1\end{array}\right)$ & Kyropoulos & $0.25-1$ \\
\#2,SK3R/45-square, 1 & $\left(\begin{array}{llll}1 & 0 & \overline{1} & 2\end{array}\right)$ & Kyropoulos & $0.25-1$ \\
\#3, SHDC15-1/20, 2 & $\left(\begin{array}{llll}1 & 1 & 2 & 0\end{array}\right)$ & HDSM & $6-10$ \\
\#4, SVA1/20, 2 & $\left(\begin{array}{llll}1 & 1 & 2 & 0\end{array}\right)$ & Verneuil & $10-15$ \\
\#5, Mono crys./24, 1 & $\left(\begin{array}{llll}0 & 0 & 0 & 1\end{array}\right)$ & $\begin{array}{l}\text { Modified } \\
\text { Kyropoulos }\end{array}$ & - \\
\hline
\end{tabular}

\subsection{Laboratory source measurements}

Double-crystal diffractometer measurements of sample \#1 reveal a FWHM of the rocking curve in symmetrical ( $\left.\begin{array}{llll}0 & 0 & 0 & 12\end{array}\right)$ reflection (Bragg angle $\theta=45.33^{\circ}$ ) of $\Delta \theta_{1 / 2}=2.4^{\prime \prime}$ whereas the peak reflectivity factor is $R=0.75$. Double-slit measurements of this reflection reveal no bending of the sample ( $\mathrm{R}>500 \mathrm{~m}$ ), see Fig. 4 . The double-crystal $\mathrm{X}$-ray topography with the $\left(\begin{array}{llll}0 & 0 & 0 & 12\end{array}\right)$ reflection (see Fig. 5) reveals the structural imperfections (dislocations and microfractures) and scratch background left by the polishing process. The measurement of two topographs which correspond to opposite sides of rocking curve enables exclusion of the influence of X-ray film defects during the topographs analysis. Changing of the contrast on some elements between both topographs can help to evaluate crystalline lattice deformation. The markings present for both topographs correspond to the actual crystal imperfections whereas others are induced by the quality of the photographic film (some film artifacts are denoted by black arrows). Dislocations (exemplarily denoted by white dotted arrows), microfractures (denoted by red arrows) and
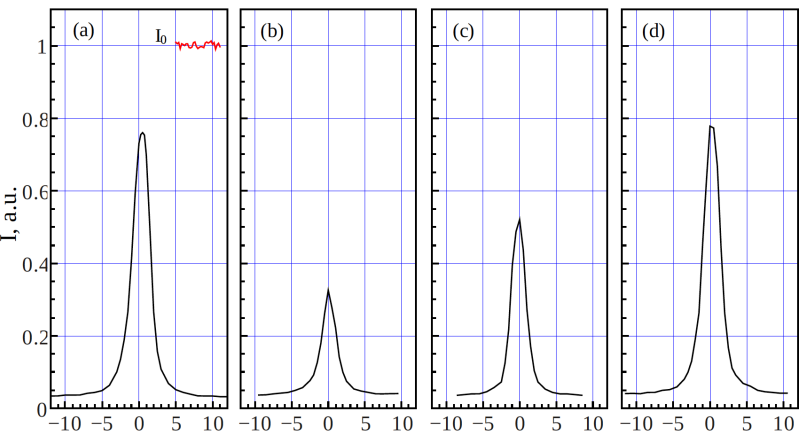

$\Delta \omega$, arcsec

Figure 4: (a) Double-crystal rocking curve of symmetrical 000 12 reflection of wafer \#1 at CuKa1 radiation. FWHM of the curve $\Delta \theta_{1 / 2}=2.4 "$, peak reflectivity $\mathrm{R}=0.75$. $\mathrm{I}_{0}$ denotes the intensity level of incident beam. Measurement regime: $16 \mathrm{kV}$, $15 \mathrm{~mA}$, detector speed limit is 104 counts/s, horizontal slit $\mathrm{H}=$ $1 \mathrm{~mm}$, vertical slit $V=1 \mathrm{~mm}$. Experimental results of wafer \#1 with the double-slit setup, for individually open slits (b) S1, (c) S2 and (d) simultaneous S1 + S2 (see Fig. 1). The FWHMs of all three peaks are equal to $\Delta \theta_{1 / 2}=2.5$ " whereas the intensity of the S1 + S2 peak is the sum of S1 and S2 intensities, thus indicating the absence of sample bending $(R>500 \mathrm{~m})$.
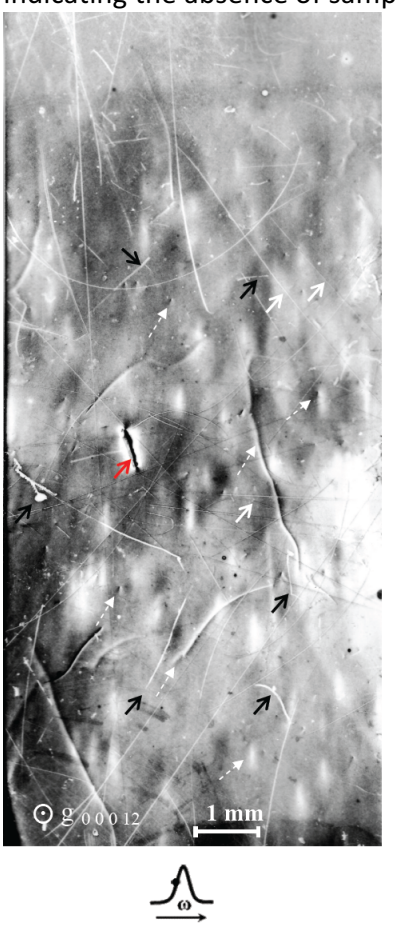

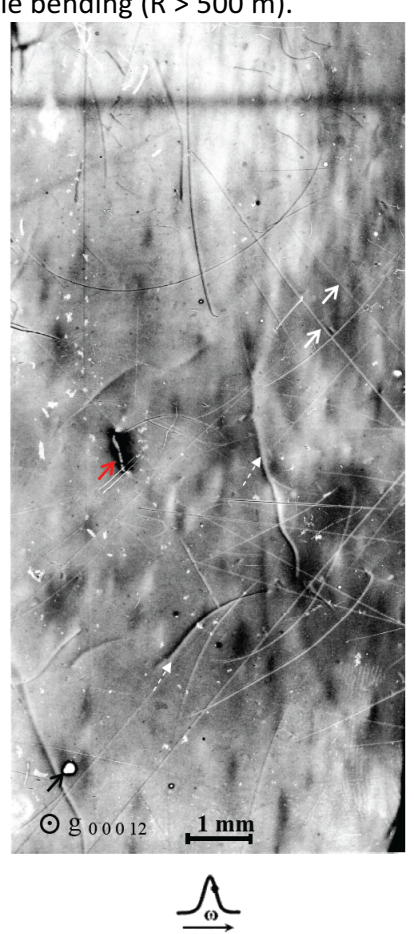

Figure 5: Double-crystal X-ray topographs of wafer \#1 measured at the opposite slopes of the rocking in symmetrical

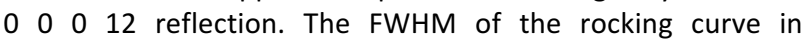
topography mode with wide incident beam is $\Delta \omega_{1 / 2}=2.8 "$. Measurement regime: $30 \mathrm{kV}, 10 \mathrm{~mA}, 30 \mathrm{~min}$ exposure. Structural imperfections (dislocations and fractures) and scratch background can be observed. Black, white, red and white dotted arrows indicate artifacts, polishing defects, microfractures and dislocations, respectively. A projection of the diffraction vector $\mathbf{g}_{00012}$ at the topograph surface is shown. 
polishing defects (thin and long non-parallel lines denoted by white arrows) can be observed on the topographs. It is difficult to determine the nature of some artifacts. In particular, the round lines with low curve radius are similar to regular dislocations but could be an artifact of image processing. The dislocation density within the wafer \#1 can be estimated to be approximately $100 \mathrm{~cm}^{-2}$.
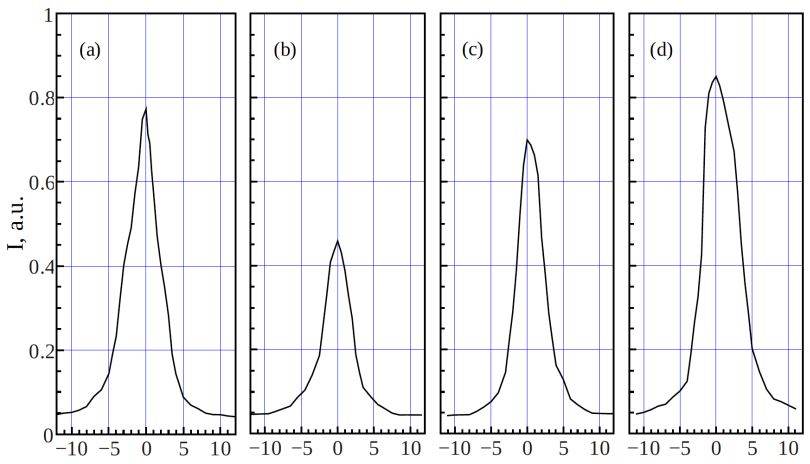

$$
\Delta \omega, \operatorname{arcsec}
$$

Figure 6: (a) Double-crystal rocking curve for the asymmetrical $13 \overline{4} 4$ reflection of wafer $\# 2$. FWHM of the curve $\Delta \omega_{1 / 2}=4.9 "$. Experimental results of wafer \#2 with the double-slit setup, cases of individually open slits (b) S1, (c) S2 and (d) simultaneous S1 + S2. The FWHM for the case of both slits open is increased from 4 " to $6 "$ ", thus bearing the evidence of a slight sample bending.
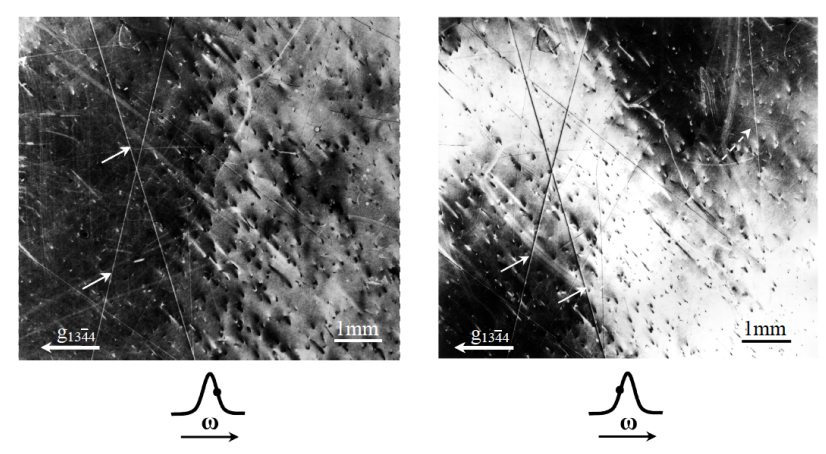

Figure 7: Double-crystal X-ray topographs of wafer \#2, measured at opposite slopes of the rocking curve in asymmetrical $13 \overline{4} 4$ reflection. The FWHM of the rocking curve in topography mode is $\Delta \omega_{1 / 2}=6 "$. Measurement regime: $30 \mathrm{kV}, 10 \mathrm{~mA}, 20$ minutes exposure. A dislocation (white dotted arrow) and scratch background (white arrows) are denoted. A projection of the diffraction vector $\mathbf{g}_{13-44}$ at the surface of topograph is shown.
Measurements of wafer \#2 at the double-crystal diffractometer show that the FWHM of the rocking curve in asymmetrical (1 $3 \overline{4} 4$ ) reflection with $\omega_{\mathrm{B}}$ diffraction geometry (Bragg angle $\theta=45.57^{\circ}$, angle between the surface and reflecting atomic planes $\varphi=18^{\circ}$, grazing angle $\omega=\theta-\varphi=27.57^{\circ}$, reflection asymmetry factor $\mathrm{b}=0.51$ ) equals $\Delta \omega_{1 / 2}=4.9^{\prime \prime}$, see Fig. 6a. An estimation for the symmetrical reflection $\left(\begin{array}{llll}0 & 0 & 0 & 12\end{array}\right)$ gives $\omega_{\mathrm{s}}=\omega \cdot \sqrt{\mathrm{b}}=3.5 "$. In the double-slit measurements a slight sample deformation was detected, however the registered peaks are not split, see Fig. $6 \mathrm{~d}$. When both slits are open the FWHM of the rocking curve is increased from 4" (for slits $S_{1}$ or $S_{2}$ ) to 6", indicating a slight curvature of the sample close to $\mathrm{R} \sim 500 \mathrm{~m}$, however the topography images are still non-uniform, see Fig. 7. The double-crystal X-ray topography for the (1 $3 \overline{4} 4$ ) reflection, see Fig. 7, reveals the presence of dislocations partially grouped within shear bands (exemplarily denoted by a white dotted arrows) and scratch background left by polishing (denoted by white arrows), scratches that indicates the insufficient removal depth during the chemical-mechanical treatment. The dislocation density within the wafer $\# 2$ can be estimated to be $\sim 10^{3}-10^{4} \mathrm{~cm}^{-2}$.

\subsection{Synchrotron radiation measurements}

Several white beam topographs of wafer \#1 are presented in Fig. 8. The average dislocation density is about 100-1000 $\mathrm{cm}^{-2}$. As it can be seen from the topographs of the whole wafer (Fig.8-a) less dislocations are detected in the left wafer side. This was observed for several different reflections and reveals the lower concentration of defects. The dislocation density in this area is about $100 \mathrm{~cm}^{-2}$. Topographs with several reflections from one particular region are depicted in Fig. $8 \mathrm{~b}-\mathrm{f}$ and details of four regions for one particular reflection are shown in Fig. 8-g.

The white beam topograph of the wafer \#2 (SK3R) in Fig. 9-a reveals a homogeneous distribution of

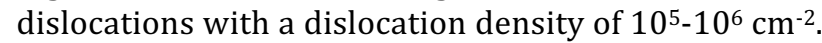
Results from the other wafers (not shown) grown by the same technique and orientation show the same feature of a homogenous distribution of very high dislocation density across the whole wafer. A topograph of the wafer \#3 (SHDC15-1) grown by HDSM with growth direction of $\left(\begin{array}{llll}1 & 1 & \overline{2} & 0\end{array}\right)$ is depicted in Fig. 9-b. A homogeneous distribution of dislocations with an estimated density of more than $10^{5}-10^{6} \mathrm{~cm}^{-2}$ is observed. 
Fig. 10-a shows results from wafer \#4 grown by the Verneuil technique and along the $C$ plane. Investigation of several reflections (not shown) confirmed the presence of small angle grain boundaries in this wafer. The dislocation density is so high $\left(>10^{6} \mathrm{~cm}^{-2}\right)$ that no single dislocation can be resolved. Very strong strain is evidenced by the deformation of the image, with some topographs appearing as split images that result from diffraction from grain boundaries.

Fig. 10-b presents the topograph of wafer \#5, one of the best commercial available crystals and reveals a high dislocation density, more than $10^{5}-10^{6} \mathrm{~cm}^{-2}$, in contrast to estimated dislocation densities from "Monocrystal", Stavropol, of better than $10^{3} \mathrm{~cm}^{-2}$. This large difference demonstrates how much more stringent the constraints are for x-ray rather than visible light optics applications. There is a homogeneous distribution of different types of straight and curved dislocations across this wafer. In small regions the dislocation tangles become less dense.

The quality of our best sapphire (wafer \#1) was further assessed by backscattering high-resolution topography where the spectral reflectivity was recorded with an incoming beam of $0.7 \mathrm{meV}$ bandwidth. These results are encouraging, as the intrinsic sapphire resolution function is $<2 \mathrm{meV}$ for this batch of crystals, albeit only over a small area. However, the surface of this small area exceeds the available area in the crystal reported in Ref. [13] by almost one order of magnitude.

\section{Discussion}

Topography results show that dislocations are the only type of defects in all sapphire wafers grown by the Kyropoulos technique and by the HDSM. No stacking faults were observed in the crystals grown by all used techniques except highly strained Verneuil grown crystals that also exhibit small angle grain boundaries. Heat exchange method grown crystals, not investigated herein, showed similar dislocations in white beam topography [18].

In general, dislocations appear in different shapes. They are mainly curved lines that correspond to a mixture of edge and screw dislocation types. Analysing these mixed type dislocations is very difficult because they do not easily vanish in topographs with different diffraction vectors [19]. The slowly grown crystals showed less dislocation curvature compared to those with higher growth rates.

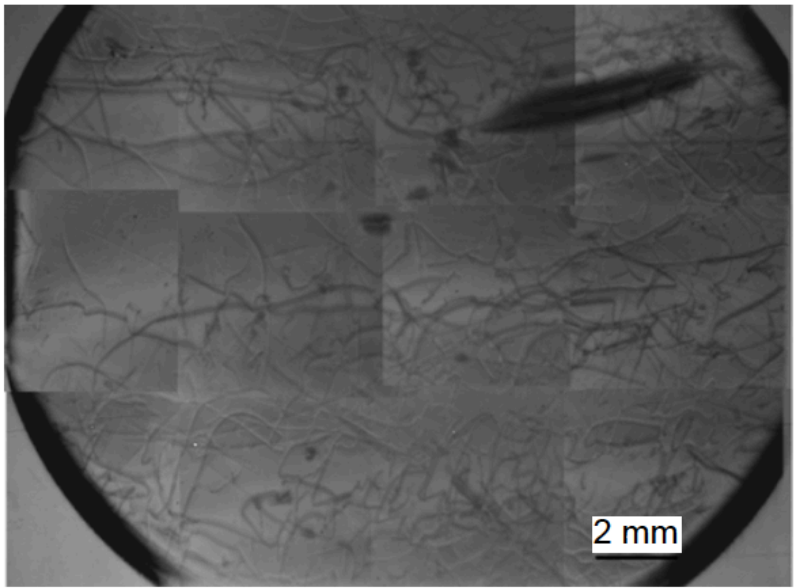

(a) Wafer \#1 (SK1C)

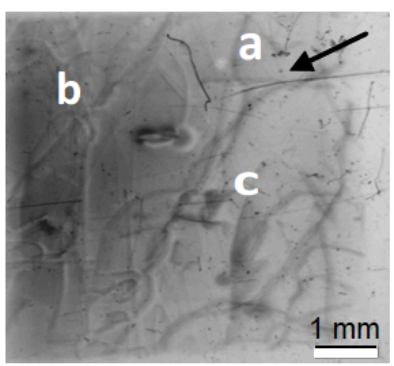

(b) -2202

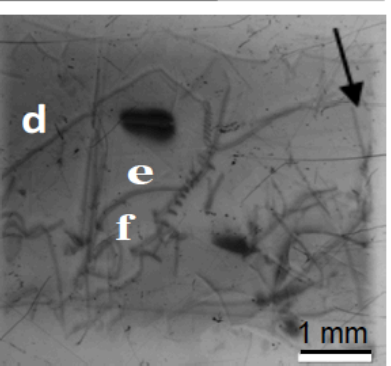

(c) $0-222$
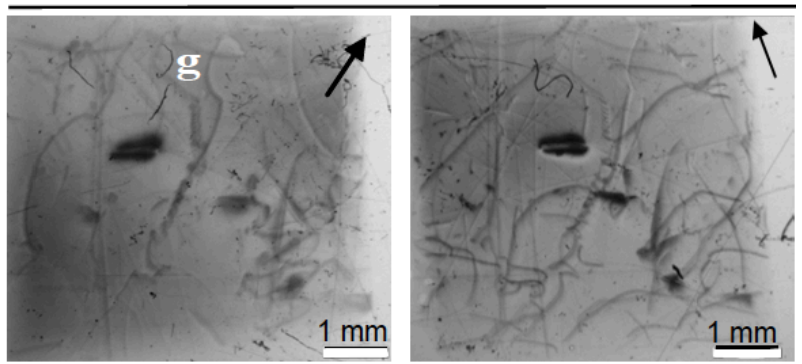

(d) $20-22$

(e) $-14-34$

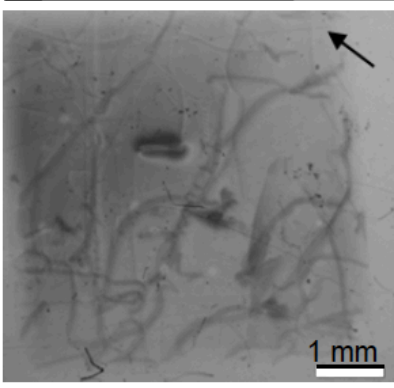

(f) $-24-23$

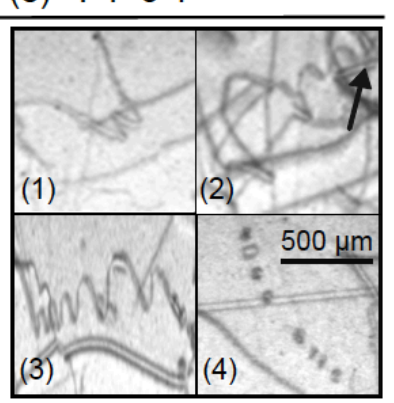

(g) $23-55$
Figure 8 (a) White-beam topograph of wafer \#1 (SK1C) at different magnification and different reflections. The reflections are: (b) $\overline{2} 20$ 2, (c) $0 \overline{2} 2$ 2, (d) $20 \overline{2} 2$, (e) $\overline{1} 4 \overline{3} 4$ (f) $\overline{2} 4 \overline{2} 3$ of same region of wafer $\# 1$. White letters denote specific dislocations, see text. (g) Three different helical dislocations $(1,2,3)$ and a series of loop dislocations (4) from a nearby region for reflection $23 \overline{5} 5$. Black arrows denote the diffraction vector, $\mathbf{g}$. 
As revealed by the topographs, dislocations appear as an open or dense tangle. This indicates that stress during growth produces knots and tangles from several single dislocations with different Burgers vectors. This could be related to fast crystal growth rate and thermal fluctuation [19].
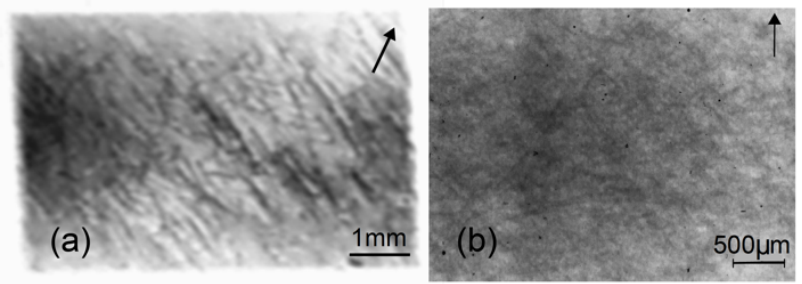

Figure 9 White-beam topograph of (a) wafer \#2 and (b) wafer \#3. Black arrows denote the diffraction vector, $\mathbf{g}$.
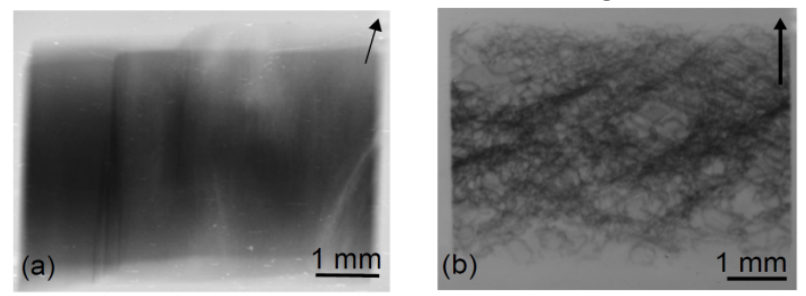

Figure 10 White-beam topograph of (a) wafer \#4 and (b) wafer \#5 . Black arrows denote the diffraction vector, $\mathbf{g}$.

Another important feature is the appearance of helically shaped dislocations, as seen in Fig. $8 \mathrm{c}$-g. These helices are another type of dislocation in sapphire which were first observed in 1971 [20]; they only appear in very high quality crystals or regions of crystals [21]. We observed them when the dislocation density is less than $10^{3} \mathrm{~cm}^{-2}$. When a screw dislocation is subjected to absorption or emission of vacancies or dopant inhomogeneity [22] they have the possibility to climb along their Burgers vector and make a helix from a straight dislocation line. Screw dislocations are more mobile than edge dislocations, thus it is easier for them to move and make turns of helices [21,22]. Fig. 8-g-(1-3) show three helical dislocations at different level of formation. The top left helical dislocation, Fig. 8-g-(1), has only two turns and seems to be at the starting point of its formation whereas Fig. 8-g-(2) and -(3) exhibit more turns. It might also be assumed that helices are unwound towards lines. In that case we expect to see changes of curved lines to straight lines too due to assumed relaxation, but as we do not observe many straight dislocations, the former idea of evolving dislocations from linear to helical has more merit. Those three helices are less compact compared to the helical dislocation $g$, shown in Fig. 8-d. This could be the result of a variation in the subjected thermal stress in different parts of the crystal. A simulation of thermal stress for the Kyropoulos grown crystal showed that near the crystallization interface, at the shouldering location, the tailing location of a boule experiences high thermal stresses [23].

There are few loop dislocations too, a series of which are shown in Fig. 8-g-(4). There are several possible ways to obtain loop shaped dislocations. One proposed idea is that helices can be a transformed to loops under special circumstances like thermal stress. But in this case we should expect very few loops, maximum three [21]. In our case we observe a row of loops which perhaps resulted from interaction of two parallel helices with opposite twist. The interactions between each two opposite turn can yield closed loops [21].

From several topography images one can determine the Burgers vector $\mathbf{b}$, the essential parameter of a dislocation. The determination of $\mathbf{b}$ can be obtained by applying the condition $\mathbf{g} \cdot \mathbf{b}=0$, where $\mathbf{g}$ is the diffraction vector and $\mathbf{b}$ is the Burger vector. Dislocations have contrast only if $\mathbf{g} \cdot \mathbf{b} \neq 0$. The screw type dislocation are invisible if $\mathbf{g} \cdot \mathbf{b}=0, \mathbf{b}$ is parallel to the dislocation line vector, $\mathbf{u}$. For edge and mixed dislocations it is invisible if conditions fulfill $\mathbf{g} \cdot \mathbf{b}=\mathbf{0}$ and $\mathbf{g} . \mathbf{b} \times \mathbf{u}=0$, where $\mathbf{b}$ is perpendicular to $\mathbf{u}$.

Here we show some selected topographs of the same region of sample \#1 which has the best quality among the studied crystals, in Fig. 8 b-f. Dislocations indicated as $a, b$ and $c$ exhibit no contrast in the reflection $0 \overline{2} 22$ and show contrast in the other reflections. In addition, we know the possible directions of dislocations and Burger vectors of dislocations in sapphire single crystals from an earlier study [18]. So the Burgers vector of $a, b$ and $c$ are parallel to [2 $\overline{1} \overline{1}$ 0]. Dislocations labeled $d, e$ and $f$ shows strong contrast in reflections $0 \overline{2} 22, \overline{1} 4 \overline{3} 4$ and $\overline{2} 4 \overline{2} 3$ but are invisible in $20 \overline{2} 2$, thus the Burgers vectors for these dislocations are parallel to $\overline{1} 2 \frac{1}{1} 0$, similar to what was suggested in Ref. [18]. The helical dislocation $g$ is invisible in $\overline{2} 202$ so $\mathbf{b}$ is parallel to [ $\left.\begin{array}{llll}1 & 1 & \overline{2} & 0\end{array}\right]$. It is known that the direction of helices is parallel to their Burgers vector and that they are edge type dislocation [21].

One can say that the Burgers vector of dislocations $a$, $b, c, d, e, f$ and $g$ belong to the $<2 \overline{1} \overline{1} 0>$ class, one of two reported Burgers vectors in this sapphire crystals [18]. As it was noted, these curved dislocations are mostly mixtures of screw and edge types. 
The crystals with the lowest dislocation density, $\sim 100 \mathrm{~cm}^{-2}$, i.e. those grown by the Kyropoulos technique, are found to have the minimal full-width at half-maximum for diffraction curves ( 2.4") among the studied batch. Furthermore, the samples produced from a crystal of this kind demonstrated a high spectral reflectivity in backscattering highresolution topography with a $0.7 \mathrm{meV}$ channel cut monochromator at energy of $23.9 \mathrm{keV}$. An influence of dislocations on the widening of diffraction peaks can be induced by the strain fields from dislocations itself as well as by the effect of dislocation polygonization that forms misoriented blocks within the crystal. It should be noted that the point imperfections in crystals also bear a negative influence: partially, the distortion of linear dislocations and formation of helicoids are possible within the media having a high concentration of point imperfections. Both high crystallization temperature $\left(2040^{\circ} \mathrm{C}\right)$ and disproportionate evaporation from the fusion mixture to the vacuum are contributing to the formation of those imperfections.

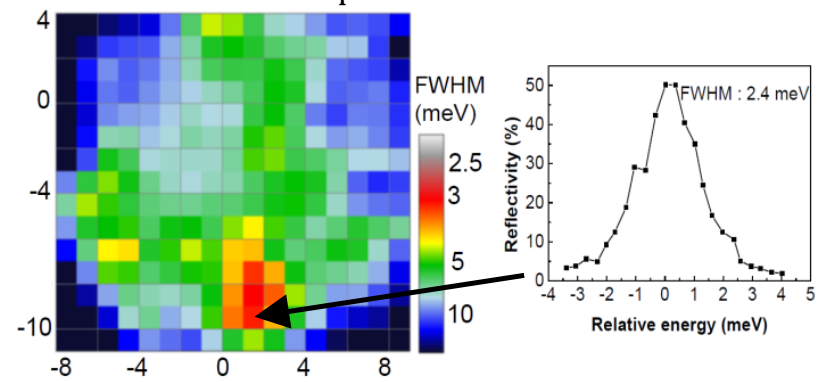

Figure 11: (left) Map of the resolution function width of a sapphire grown by the Kyropoulos Technique (wafer \#1). Sapphire dimensions are in $\mathrm{mm}$. (right) Backscattering spectral reflectivity measured at $24 \mathrm{keV}$ at the best spot indicated by the arrow.

A low dislocation density in the sapphire crystals grown by Kyropoulos technique in comparison with the ones grown by Czochralski and Stepanov technique have been reported previously [24]. It is thus reasonable to assume that the definitive factors in suppressing the formation of blocks and dislocations in crystals [12] grown by Kyropoulos technique are the relatively low temperature gradients within the hot zone $\left(10-20{\mathrm{~K} . \mathrm{cm}^{-1}}^{-1}\right.$ and the absence of contact between the growing crystal and the walls of a fusion container. Hence, it is planned to conduct any subsequent experiments on sapphire crystal growth with application of the Kyropoulos technique to reduce the dislocation density and concentration of other imperfections.

\section{Conclusion}

X-ray investigations of the real structure in sapphire have been performed for a batch of crystals synthesized by almost all known growth techniques. The highest quality sapphire for x-ray optics applications are found to be those grown by the Kyropoulos method, with a FWHM of the rocking curve of $\Delta \theta_{1 / 2}=2.4$ " in 00012 reflection of wafer \#1. The other wafers all showed higher FWHM and are unsuitable for high quality x-ray optics, at least with standard growth parameters. White beam topography of several wafers showed that the main defect type is dislocations, which appear as straight line, curves, and helical shapes. We could identify a Burger vector of $<2 \overline{1} \overline{1} 0>$ using dislocation contrast criteria for some of the dislocations. The average dislocation densities are linked to the growth techniques and pulling rates. Studies on several wafers grown by same technique showed similar features and dislocation densities. For the best crystal grown by the Kyropoulos technique, close to ideal $\mathrm{x}$ ray reflectivity is observed in backscattering mode at $24 \mathrm{keV}$ over an area $>1 \mathrm{~mm}^{2}$.

\section{Acknowledgements.}

The Helmholtz Association of German Research Center and the Russian ministry of science and education are acknowledged for the HelmholtzRussia Joint Research Group under grant HRJRG-402 and RFMEFI62114X0005, respectively. RPH acknowledges support from the Materials Sciences and Engineering Division, Office of Basic Energy Sciences, US Department of Energy. Provision of synchrotron radiation beam time at TOPO-TOMO, ANKA, Karlsruhe and P01, Petra III, DESY, Hamburg is gratefully acknowledged. Monocrystal, Stavropol, Russia is acknowledged for provision of a commercial crystal. Jürgen Härtwig, Ben Larson, Gene Ice and Tom Watkins are acknowledged for helpful discussions.

\section{References}

[1] M. Hart, L. Berman Acta Crystallogr. A 54, 850-858 (1998).

[2] E. Ziegler, T. Bigault, and J. Hoszowska, Synchrotron Radiation Instrumentation, AIP Proceedings 705, 768-771 (2004).

[3] J. Hrdý and O. Pacherová, Nucl. Instr. Meth. Phys. Res. A 327, 605-611 (1993). 
[4] C. David, J. Bruder, T. Rohbeck, C. Grünzweig, C. Kottler, A. Diaz, O. Bunk, and F. Pfeiffer, Microelectron. Eng. 84, 1172-1177 (2007).

[5] T.S. Toellner, Hyperfine Interact. 125, 3-28 (2000).

[6] Yu. V. Shvyd'ko, X-ray Optics: High-Energy-Resolution Applications (Springer, Berlin, 2004).

[7] Yu. V. Shvyd'ko and E. Gerdau, Hyperfine Interact. 123, 741-776 (1999).

[8] Yu. V. Shvyd'ko, M. Gerken, H. Franz, M.Lucht, and E. Gerdau, Europhys. Lett. 56, 309-315 (2001).

[9] Kh. S. Bagdasarov, High-temperature crystallization from melt (Fismatlit, Moscow, 2004) p. 160.

[10] V.E. Asadchikov, A.V. Butashin, Y.O. Volkov, Yu. V. Grischenko, A. N. Deryabin, M. L. Zanaveskin, V. M. Kanevskii, I. V. Kozhevnikov, B. S. Roschin, E. O. Tikhonov, A. L. Tolstikhina, and V. A. Fedorov, Inorganic Materials. 45, 1635-1639 (2009).

[11] J. Cui, A. Sun, M. Reshichkov, F. Yun, A. Baski, and H. Morkoç, MRS Internet J. Nitride Semicond. Res. 5, 1-6 (2000).

[12] V.E. Asadchikov, A.V. Butashin, V.M. Kanevsky, A.E. Muslimov, and B.S. Roshchin, Sapphire: Structure, Technology, and Application, (Nova Science Publishers. New York, 2013) pp. 35-73.

[13] I. Sergueev, H.-C. Wille, R. P. Hermann, D. Bessas, Y. V. Shvyd'ko, M. Zajac, and R. Rüffer, J. Synchrotron Rad. 18, 802-810 (2011).
[14] B. Klobes, A. Desmedt, I. Sergueev, K. Schmalzl, and R. P. Hermann, EPL 103, 36001 (2013).

[15] A. Rack, T. Weitkamp, S. Bauer Trabesi, P. Modregger, A. Cecilia, T. dos Santos Rolo, T. Rack, D. Haas, R. Simon, T. Baumbach, R. Heldele, M. Schulz, B. Mayzel, A. N. Danilewsky, T. Waterstradt, W. Diete, H. Riesemeier and B. R. Müller, Nuclear Instruments and Methods, NIM-B 267, 1978-1988 (2009).

[16] X. R. Huang, J. Appl. Cryst. 43, 926 (2010).

[17] Sutter, J. P., Baron, A. Q. R., Miwa, D., Nishino, Y., Tamasaku, K. \& Ishikawa, T., J. Synchrotron Rad. 13, 278280 (2006).

[18] W.M. Chen, P.J. McNally, Yu.V. Shvyd'ko, T. Tuomic, A.N. Danilewsky, and M. Lerche, J. Cryst Growth 252, 113-119 (2003).

[19] J. L. Caslavsky, C. P. Gazzara, and R. M. Middleton, Philos. Mag. 25, 35-44 (1972).

[20] J. L. Caslavsky and C. P. Gazzara, J. Mater. Sci. 6, 1-4 (1971).

[21] J. L. Caslavsky and C. P. Gazzara, Philos. Mag. 26, 961-975 (1972).

[22] J, Weertman, Phys. Rev. 107, 1259- 1261 (1957).

[23] X. Chenghai, M. Songhe, Z. Mingfu, Z. Hongbo, and W. Guigen, Chinese Journal of Aeronautics 20, 475-480 (2007).

[24] D.C. Harris, Proc. SPIE 7302, 7302021-73020212 (2009). 